\title{
Spin-resolved impurity resonance states in electron-doped cuprate superconductors
}

\author{
Bin $\mathrm{Liu}^{* 1}$ \\ ${ }^{1}$ Max-Planck-Institut für Physik komplexer Systeme, D-01187 Dresden, Germany
}

\begin{abstract}
With the aim at understanding the non-monotonic $d_{x^{2}-y^{2}}$-wave gap, we analyze the local electronic structure near impurities in the electron-doped cuprate superconductors. We find that the local density of states near a non-magnetic impurity in the scenario of $d_{x^{2}-y^{2}}$-wave superconductivity with higher harmonics is qualitatively different from that obtained from the $d_{x^{2}-y^{2}}$-wave superconductivity coexisting with antiferromagnetic spin density wave order. We propose that spinpolarized scanning tunneling microscopy measurements can distinguish the two scenarios and shed

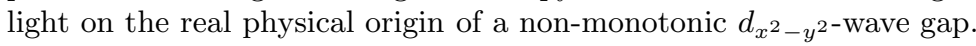

PACS numbers: 74.72.Jt, 74.20.Mn, 74.20.Rp, 74.25.Jb

Pairing symmetry in the electron-doped cuprate hightemperature superconductors has been extensively studied experimentally and theoretically. In contrast to the

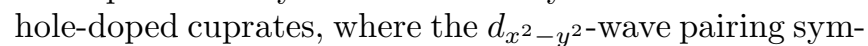
metry has been generally accepted ${ }^{1,2}$, the earlier point contact tunneling spectra suggested an s-wave like symmetry due to the absence of zero bias conductance peak in the spectrum ${ }^{3} \cdot \underline{4}$. Recently, the phase sensitive scanning (SQUID) measurements $\underline{\underline{5}}$, nuclear magnetic resonance study $\underline{\underline{6}}$, and angle-resolved photoemission spectroscopy (ARPES) experiments 7,8 have provided strong evidences that the electron-doped cuprates are also the $d_{x^{2}-y^{2}}$-wave superconductors. However, the functional form of the $d_{x^{2}-y^{2}}$-wave gap in electron-doped materials is a more subtle issue. A non-monotonic $d_{x^{2}-y^{2} \text {-wave }}$ gap with a maximal value in between nodal and antinodal points on the Fermi surface (FS) has been measured in Raman experiments in $\mathrm{NCCO}^{9}$ and ARPES data on the leading-edge gap in $\operatorname{Pr}_{0.89} \mathrm{LaCe}_{0.11} \mathrm{CuO}_{4}{ }^{7}$.

Up to now, the physical origin of such non-monotonic $d_{x^{2}-y^{2}}$-wave gap is still under debate. Two kinds of theoretical explanations have been put forward. One is to extend the superconducting (SC) gap out of the simplest $d_{x^{2}-y^{2}}$-wave via the inclusion of higher harmonics.2. Based on the theoretical assumption, the $d_{x^{2}-y^{2}}$-wave pairing is caused by the attractive interaction with the continuum of overdamped antiferromagnetic (AF) spin fluctuations, which generates a maximal gap near the hot spots (the points along the FS separated by the $\mathrm{AF}$ move vector $Q_{A F}$ ). Since the hot spots in the optimally doped NCCO and PCCO are located close to Brillouin zone diagonals, one can generally expect the $d_{x^{2}-y^{2}}$-wave gap to be non-monotonic. The other one is the coexisting scenario in which the AF long-range order coexists with the $d_{x^{2}-y^{2}}$-wave order ${ }^{10,11}$. The neutron scattering 12 and transport experiments 13 have observed a robust AF order, which survives a broad doping region in the phase diagram. On the other hand, the ARPES measurements revealed the intriguing doping evolution of the FS in $\mathrm{NCCO}^{14}$, where two inequivalent pockets around $(\pi, 0)$ and $(\pi / 2, \pi / 2)$ shown in the FS have been explained to the band folding due to the $\mathrm{AF}$ order $\underline{15}$. As a consequence, the resulting quasiparticle excitation can be gapped by both orders, and the non-monotonic

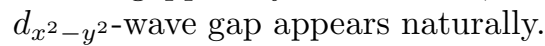

In this paper, we argue that the local electronic structure near impurities can provide important insight into

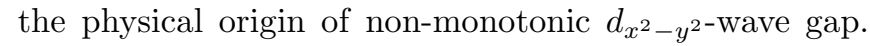
We calculate local density of states (LDOS) around a non-magnetic impurity starting from two scenarios: $d_{x^{2}-y^{2}}$-wave superconductivity with a higher harmonic versus $d_{x^{2}-y^{2}}$-wave superconductivity coexisting with $\mathrm{AF}$ spin density wave (SDW) order. We find that the behavior of density of states (DOS) in both scenarios suggests

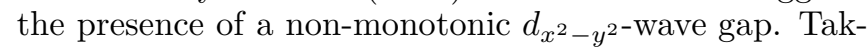
ing the single non-magnetic impurity into account, we find that in the scenario of $d_{x^{2}-y^{2}}$-wave superconductivity with a higher harmonic, the LDOS behaves similar to that shown in hole-doped cuprates $\frac{16}{}$, i.e. a single resonance state near Fermi energy appears at impurity site. However, due to introducing AF SDW order in the latter scenario, the LDOS indicates a spin-resolved feature, i.e. two resonance states occur at impurity site with different energies. For the sufficiently large SDW order, one spin component (spin-up or spin-down) turns out to be dominant, and although the DOS shows a U-shaped behavior, the presence of resonance states at low energies in LDOS rules out the possibility of s-wave pairing symmetry $3,4,17,18$. Thus, we conclude that the different electronic structure near a non-magnetic impurity can differentiate between above scenarios and can be checked by the further scanning tunneling microscopy (STM) experiments.

We start from a phenomenological superconducting Hamiltonian $H_{S C}=\sum_{\mathbf{k} \sigma}\left[\xi_{\mathbf{k}} c_{\mathbf{k} \sigma}^{\dagger} c_{\mathbf{k} \sigma}+\Delta_{\mathbf{k}}\left(c_{\mathbf{k} \uparrow}^{\dagger} c_{-\mathbf{k} \downarrow}^{\dagger}+\right.\right.$ $\left.\left.c_{-\mathbf{k} \downarrow} c_{\mathbf{k} \uparrow}\right)\right]$, where $c_{\mathbf{k} \sigma}^{\dagger}\left(c_{\mathbf{k} \sigma}\right)$ is the fermion creation (destruction) operator for an electron in the state with wave vector $\mathbf{k}$ and spin projection $\sigma$, and $\xi_{\mathbf{k}}=\varepsilon_{\mathbf{k}}-\mu$ with the normal state tight binding dispersion $\varepsilon_{\mathbf{k}}=-2 t\left[\cos \left(k_{x}\right)+\cos \left(k_{y}\right)\right]-4 t_{1} \cos \left(k_{x}\right) \cos \left(k_{y}\right)-$ $2 t_{2}\left[\cos \left(2 k_{x}\right)+\cos \left(2 k_{y}\right)\right]-4 t_{3}\left[\cos \left(2 k_{x}\right) \cos \left(k_{y}\right)+\right.$ $\left.\cos \left(k_{x}\right) \cos \left(2 k_{y}\right)\right] \quad-\quad 4 t_{4} \cos \left(2 k_{x}\right) \cos \left(2 k_{y}\right) \quad$ where $\left(t, t_{1}, t_{2}, t_{3}, t_{4}, \mu\right)=(120,-60,34,7,20,-82)$ with the unit of meV at 0.11 doping 19 reproduce the underlying FS as inferred from recent ARPES experiment ${ }^{7}$. As argued above, the maximum SC gap is achieved 
near hot $\operatorname{spots}^{1,2,9}$, which are located much closer to the zone diagonal, leading to a non-monotonic behavior of the SC gap. A good fit of $\Delta_{\mathbf{k}}$ to the experimental data is achieved via the inclusion of a higher harmonic, such that $\Delta_{\mathbf{k}}=\sum_{i=1,3} \Delta_{i}\left[\cos \left(i k_{x}\right)-\cos \left(i k_{y}\right)\right] / 2$ with $\Delta_{1}=5.44 \mathrm{meV}$ and $\Delta_{3}=-2.34 \mathrm{meV}$ ensures that the maximum of $\left|\Delta_{\mathbf{k}}\right|$ along the FS is located at the hot spots. Corresponding FS and a non-monotonic gap as a function of the FS angle have been shown in Fig.4 of Ref.20.

By introducing a two-component Nambu spinor operator, $\Psi_{\mathbf{k}}=\left(c_{\mathbf{k} \uparrow}, c_{-\mathbf{k} \downarrow}^{\dagger}\right)^{\top}$, the matrix Green's function $G_{0}$ in the superconducting state can be written by

$$
G_{0}\left(\mathbf{k}, i \omega_{n}\right)=\frac{i \omega_{n} \tau_{0}+\xi_{\mathbf{k}} \tau_{2}+\Delta_{\mathbf{k}} \tau_{1}}{\left(i \omega_{n}\right)^{2}-E_{\mathbf{k}}^{2}},
$$

with $E_{\mathbf{k}}=\left(\xi_{\mathbf{k}}^{2}+\Delta_{\mathbf{k}}^{2}\right)^{1 / 2}$ the quasiparticle spectrum and $\tau_{i}$ being the Pauli spin operator. The corresponding realspace Green's function is

$$
G_{0}\left(i, j ; i \omega_{n}\right)=\frac{1}{N} \sum_{\mathbf{k}} e^{i \mathbf{k} \cdot \mathbf{R}_{\mathbf{i j}}} G_{0}\left(\mathbf{k}, i \omega_{n}\right),
$$

where $\mathbf{R}_{\mathbf{i j}}=\mathbf{R}_{\mathbf{i}}-\mathbf{R}_{\mathbf{j}}$ with $\mathbf{R}_{\mathbf{i}}$ being lattice vector. In the presence of a single-site nonmagnetic impurity of strength $U_{0}$ located at the origin $r_{i}=0$, the site dependent Green's function in term of the T-matrix approach 16 can be obtained as

$$
\begin{aligned}
G\left(i, j ; i \omega_{n}\right) & =G_{0}\left(i-j ; i \omega_{n}\right) \\
& +G_{0}\left(i ; i \omega_{n}\right) T\left(i \omega_{n}\right) G_{0}\left(j ; i \omega_{n}\right),
\end{aligned}
$$

where

$$
T\left(i \omega_{n}\right)=\frac{U_{0} \tau_{3}}{1-U_{0} \tau_{3} G_{0}\left(0,0 ; i \omega_{n}\right)} .
$$

For the d-wave (with or without a higher harmonic) pairing symmetry, one can find that the local Green's function $G_{0}\left(i, i ; i \omega_{n}\right)$ is diagonal. As a result, the diagonal T-matrix reads

$$
T_{11,22}\left(i \omega_{n}\right)=\frac{ \pm U_{0}}{1-U_{0}\left[G_{0}\left(0,0 ; \pm i \omega_{n}\right)\right]_{11}}
$$

where the plus (minus) sign denotes $T_{11}\left(T_{22}\right)$, giving rise to a particle- $\left(\omega_{\text {res }}<0\right)$ and hole-like $\left(\omega_{\text {res }}>0\right)$ resonance state. These resonance states generate the sharp peaks in the LDOS only in the unitary limit $\left(\left|\omega_{\text {res }}\right| / \Delta_{1} \leq 1\right)$ where $1=U_{0} R e\left[G_{0}\left(0,0 ; \pm \omega_{\text {res }}\right)\right]_{11}$.

Finally, the LDOS which can be measured in the STM experiment is expressed as

$$
N(r, \omega)=\sum_{\sigma} N_{\sigma}(r, \omega)
$$

with spin-resolved LDOS

$$
\begin{aligned}
& N_{\uparrow}(r, \omega)=-\frac{1}{\pi} \operatorname{Im} G_{11}\left(r, r ; \omega+i 0^{\dagger}\right), \\
& N_{\downarrow}(r, \omega)=\frac{1}{\pi} \operatorname{Im} G_{22}\left(r, r ;-\omega-i 0^{\dagger}\right) .
\end{aligned}
$$

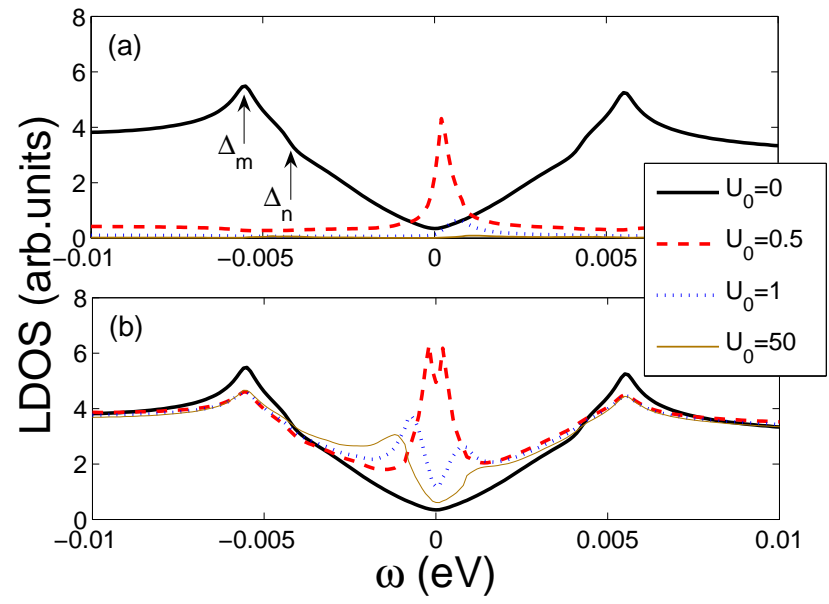

FIG. 1: (Color online) The dependence of LDOS on scattering strength $U_{0}$ (a) at impurity site and (b) on the impurity's nearest-neighbor site. $\Delta_{n}$ and $\Delta_{m}$ denote the antinodal gap and maximum gap at the hot spot respectively.

The above equations allow a complete solution as long as the order-parameter relaxation can be ignored.

In Fig.1 we present the dependence of LDOS on scattering strength $U_{0}$. The LDOS with $U_{0}=0$ (thick solid line) which is equivalent to DOS in the clean system, shows two van Hove singularities at corresponding antinodal gap $\left(\Delta_{n}\right)$ and maximum gap at the hot spot $\left(\Delta_{m}\right)$, indicating the presence of a non-monotonic $d_{x^{2}-y^{2}}$-wave gap, and is qualitatively consistent with the recent doping dependence of tunneling spectrum in $\mathrm{Pr}_{2-x} \mathrm{Ce}_{x} \mathrm{CuO}_{4-\delta}{ }^{21}$. In the presence of a non-magnetic impurity, a single resonance state appears at the impurity site (Fig.1(a)). With increasing $U_{0}$, the position of the resonance state shifts to positive high energy; meanwhile the spectral weight gradually reduces (the LDOS in Fig.1(a) with $U_{0}=50 \mathrm{eV}$ (thin solid line) has been amplified 500 times) and finally vanishes in the limit $U_{0} \longrightarrow \propto$. In the LDOS on the impurity's nearestneighbor site (Fig.1(b)), there are two resonance states locating at the positive and negative energy with different spectral weight due to the particle-hole asymmetry. These features can be understood from the Eq. (3) and Eq. (5), where the correction to $G\left(i, i ; i \omega_{n}\right)$ due to impurity scattering reads

$$
\begin{aligned}
\delta G_{11}\left(i ; i \omega_{n}\right) & =\frac{U_{0}\left[G_{0}\left(i ; i \omega_{n}\right)\right]_{11}^{2}}{1-U_{0}\left[G_{0}\left(i ; i \omega_{n}\right)\right]_{11}} \\
& -\frac{U_{0}\left[G_{0}\left(i ; i \omega_{n}\right)\right]_{12}^{2}}{1-U_{0}\left[G_{0}\left(i ;-i \omega_{n}\right)\right]_{11}} .
\end{aligned}
$$

At the impurity site, the fact $\left[G_{0}\left(i ; i \omega_{n}\right)\right]_{12}=0$ leads to a single resonance state; while on the impurity's nearestneighbor site, spectral weight of both resonance states is nonzero, i.e. $\left[G_{0}\left(i ; i \omega_{n}\right)\right]_{11,12} \neq 0$ give rise to two resonance states. Due to the same spin component at sin- 
gle site, the spin-resolved LDOS $\left(N_{\uparrow}\right.$ and $\left.N_{\downarrow}\right)$ degenerates, resulting in a degenerate single resonance state at the impurity site and two resonance states on the impurity's nearest-neighbor site in total LDOS. These features, which are qualitatively similar to the $d_{x^{2}-y^{2} \text {-wave }}$ hole-doped cuprates $\frac{16}{}$, indicate that the inclusion of a higher harmonic in the gap function basically can not alter the local electronic structure near a non-magnetic impurity, although it generates a non-monotonic $d_{x^{2}-y^{2}}$ wave gap in electron-doped cuprate superconductors.

We now compare the above results with those resulted from the coexisting AF SDW and SC phase. It is convenient to introduce a $4 \times 4$ matrix formulation, take four-component Nambu spinor $\varphi_{\mathbf{k}}=$ $\left(c_{\mathbf{k} \uparrow}, c_{\mathbf{k}+\mathbf{Q} \uparrow}, c_{-\mathbf{k} \downarrow}^{\dagger}, c_{-\mathbf{k}-\mathbf{Q} \downarrow}^{\dagger}\right)^{\top}$ with $\mathbf{Q}=(\pi, \pi)$ being the nesting vector, and then write the mean-field Hamiltonian as $H_{S C+S D W}=\sum_{\mathbf{k}} \varphi_{\mathbf{k}}^{+}\left(\xi_{\mathbf{k}} \tau_{3} \rho_{0}+M \tau_{1} \rho_{0}+\right.$ $\left.\Delta_{\mathbf{k}} \tau_{3} \rho_{1}\right) \varphi_{\mathbf{k}}$, where $\tau_{3} \rho_{1}=\left(\begin{array}{cc}0 & \tau_{3} \\ \tau_{3} & 0\end{array}\right), M$ is AF SDW order parameter and $\Delta_{\mathbf{k}}=\Delta_{1}\left[\cos \left(k_{x}\right)-\cos \left(k_{y}\right)\right] / 2$ is monotonic $d_{x^{2}-y^{2}}$-wave SC gap. Note that from now on the wave vector $\mathbf{k}$ is restricted to the magnetic Brillouin zone (MBZ).

The relevant matrix Green's function is obtained as

$$
g_{0}^{-1}\left(\mathbf{k}, i \omega_{n}\right)=i \omega_{n}-\xi_{\mathbf{k}} \tau_{3} \rho_{0}-M \tau_{1} \rho_{0}-\Delta_{\mathbf{k}} \tau_{3} \rho_{1} .
$$

To solve for the resonance state in the coexisting $\mathrm{AF}$ SDW and SC phase, we define the $2 \times 2$ Green's function as

$$
\begin{aligned}
G_{0}\left(i, j ; i \omega_{n}\right) & =\frac{1}{N} \sum_{\mathbf{k}} e^{i \mathbf{k} \cdot \mathbf{R}_{\mathbf{i j}}} \\
& \times\left(\begin{array}{cc}
G_{0}^{1}\left(\mathbf{k}, i \omega_{n}\right) & G_{0}^{2}\left(\mathbf{k}, i \omega_{n}\right) \\
G_{0}^{3}\left(\mathbf{k}, i \omega_{n}\right) & G_{0}^{4}\left(\mathbf{k}, i \omega_{n}\right)
\end{array}\right),
\end{aligned}
$$

where

$$
\begin{aligned}
G_{0}^{\mathbf{I}}\left(\mathbf{k}, i \omega_{n}\right) & =e^{-i \mathbf{Q} \cdot \mathbf{R}_{\mathbf{j}}}\left[g_{0}\right]_{\mathbf{I}}^{12}\left(\mathbf{k}, i \omega_{n}\right)+e^{i \mathbf{Q} \cdot \mathbf{R}_{\mathbf{i}}}\left[g_{0}\right]_{\mathbf{I}}^{21}\left(\mathbf{k}, i \omega_{n}\right) \\
& +e^{i \mathbf{Q} \cdot \mathbf{R}_{\mathbf{i j}}}\left[g_{0}\right]_{\mathbf{I}}^{22}\left(\mathbf{k}, i \omega_{n}\right)+\left[g_{0}\right]_{\mathbf{I}}^{1}\left(\mathbf{k}, i \omega_{n}\right), \quad(12)
\end{aligned}
$$

with $\mathbf{I}=1,2,3,4$ denoting the left-top, right-top, left-bottom and right-bottom $2 \times 2$ block element of $g_{0}\left(\mathbf{k}, i \omega_{n}\right)$. Applying T-matrix approach $\frac{16}{}$, we can easily get the LDOS in the presence of a non-magnetic impurity.

The dependence of LDOS on scattering strength $U_{0}$ for different AF SDW order $M$ is plotted in Fig.2. Following the discussions in Ref.19, we take the independent particle dispersion $\xi_{\mathbf{k}}$, and consider the doping dependent $\mathrm{AF}$ SDW order $M$. We in the following calculation choose the self-consistent value $M=0.14 \mathrm{eV}$ at 0.11 doping 19 , and the decreasing $M$ values corresponding to the doping increasing .

Before considering the effect of the impurity we briefly review the evolution of DOS with AF SDW order in the SC state $\underline{\underline{20}}$. In Fig. 2 the LDOS with $U_{0}=0$ (thick solid line) is equivalent to DOS in the clean system. In the limit $M=0 e V$, as seen in hole-doped cuprates $\frac{16}{}$, the

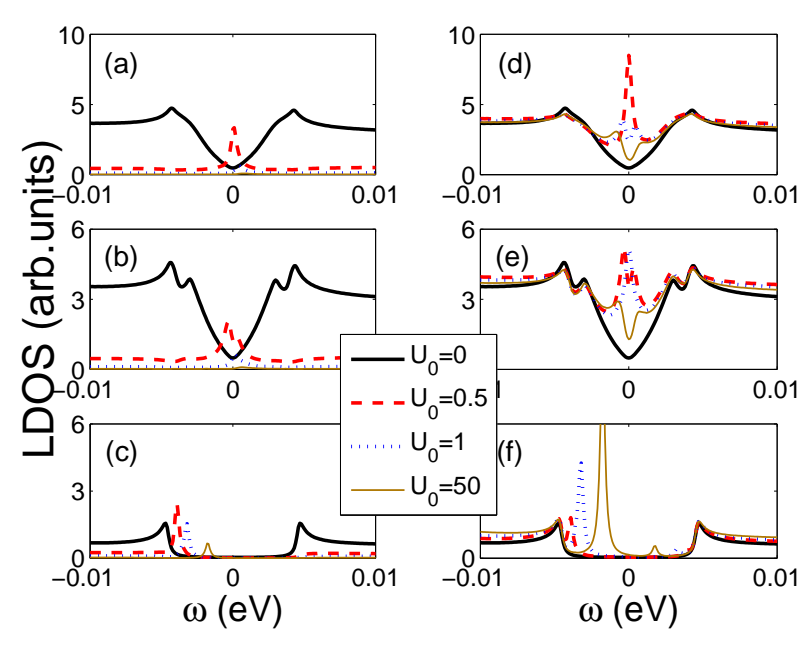

FIG. 2: (Color online) The dependence of LDOS on scattering strength $U_{0}$ for different AF SDW order $M$. LDOS at impurity site: (a) $M=0 \mathrm{eV}$, (b) $M=0.05 \mathrm{eV}$, and (c) $M=$ $0.14 \mathrm{eV}$; and LDOS on the impurity's nearest-neighbor site: (d) $M=0 \mathrm{eV}$, (e) $M=0.05 \mathrm{eV}$, and (f) $M=0.14 \mathrm{eV}$.

DOS (thick solid line in Fig.2(a)) at low energies behaves to be $\mathrm{V}$-shaped like with a monotonic $d_{x^{2}-y^{2} \text {-wave }}$ SC gap, and a coherent peak locates at the maximal gap edge. After introducing AF SDW order, another coherent peak appears at the energy less than the maximum gap (thick solid line in Fig.2(b)). Thus, a non-monotonic

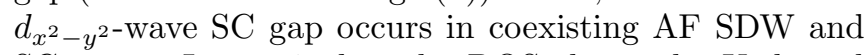
$\mathrm{SC}$ state. In particular, the DOS shows the U-shaped behavior at sufficiently large SDW order $M=0.14 \mathrm{eV}$ with doping $x=0.11^{19}$ (thick solid line in Fig.2(c)), which has been observed in earlier point contact tunneling spectra ${ }^{3.4}$. These unusual evolutions of DOS with AF SDW order are qualitatively similar to the doping evolution of DOS프, and have been explained as the result of the coexisting AF SDW and SC state ${ }^{11,20}$.

We proceed to analyze the dependence of LDOS on scattering strength $U_{0}$ near a non-magnetic impurity. For the limit AF SDW order $M=0 \mathrm{eV}$, we show that the LDOS at the impurity site (Fig.2(a)) and on the impurity's nearest-neighbor site (Fig.2(d)) are similar to that obtained from the $d_{x^{2}-y^{2} \text {-wave hole-doped }}$ superconductors 16 . With increasing AF SDW order, the LDOS at the impurity site are qualitatively different. In Fig.2(b), it is clearly shown that two resonance states at the impurity site occur near the Fermi energy, which indicates that the degenerate single resonance state with $M=0$ has separated due to the presence of AF SDW order. In principle, the spin-resolved LDOS should give rise to multiple resonance states on the impurity's nearestneighbor site, though they are actually not easy to be resolved in Fig.2(e) because of the resonance states crossing each other near Fermi energy. At sufficiently large SDW order $M=0.14 \mathrm{eV}$, one resonance state at the impurity site (Fig.2(c)) exists and shifts towards the gap edge, the 

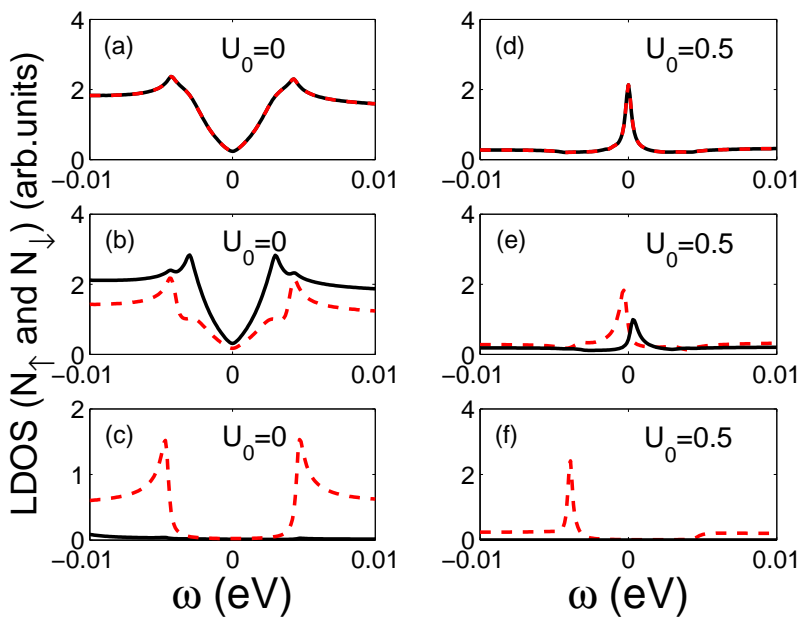

FIG. 3: (Color online) The spin-resolved LDOS $N_{\uparrow}$ (dashed line) and $N_{\downarrow}$ (solid line) at impurity site without scattering $U_{0}=0 \mathrm{eV}$ for AF SDW gap (a) $M=0 \mathrm{eV}$, (b) $M=0.05 \mathrm{eV}$, and (c) $M=0.14 \mathrm{eV}$; and with $U_{0}=0.5 \mathrm{eV}$ for (d) $M=0 \mathrm{eV}$, (e) $M=0.05 \mathrm{eV}$, and (f) $M=0.14 \mathrm{eV}$.

other one is barely visible due to the vanishing spectral weight. For the better understanding of such important features, the spin-resolved LDOS $\left(N_{\uparrow}\right.$ and $\left.N_{\downarrow}\right)$ at impurity site without scattering $U_{0}=0 \mathrm{eV}$ and with scattering strength $U_{0}=0.5 \mathrm{eV}$ are shown in Fig.3 for increasing $M$. When $M=0$, the spin-resolved $N_{\uparrow}$ and $N_{\downarrow}$ with $U_{0}=0 \mathrm{eV}$ degenerate (Fig.3a), the resulting resonance state in spin-resolved LDOS $N_{\uparrow}$ and $N_{\downarrow}$ with $U_{0}=0.5 \mathrm{eV}$ are located at the same resonance energy, leading to a degenerate single resonance state (Fig.3d). With $M$ increasing, the LDOS $N_{\uparrow}$ is not equal to $N_{\downarrow}$ (Fig.3b), thus the degenerate spin-resolved LDOS separates, leading to two resonance states at impurity site with different spectral weight (Fig.3e). At sufficiently large SDW order M, the LDOS $N_{\uparrow}$ is dominant over $N_{\downarrow}$ (Fig.3c), thus one single resonance state from spin-up component exists and shifts towards the gap edge due to the U-shaped DOS (Fig.3f), and the other one from spin-down component is barely visible due to the vanishing spectral weight (solid line in Fig.3c). Therefore due to the different spin components induced by the presence of AF SDW gap, an existing impurity will be polarized by a local net spin-up or spin-down, which leads to the splitting of the LDOS. These unique features do not appear in the scenario of

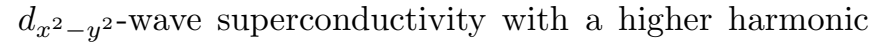
and should be detected by the spin-polarized STM measurement.

In summary, we analyze the LDOS around a nonmagnetic impurity in electron-doped cuprate superconductors starting from two different scenarios: $d_{x^{2}-y^{2}}$ wave superconductivity with a higher harmonic versus

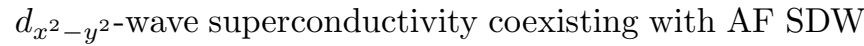
order. We find that in both cases the DOS indicates the presence of non-monotonic $d_{x^{2}-y^{2}}$-wave gap, qualitatively consistent with the recent tunneling spectrum measurement in $\operatorname{Pr}_{2-x} \mathrm{Ce}_{x} \mathrm{CuO}_{4-\delta}$ 21 , therefore both of them have been thought to be the possible physical origins of the non-monotonic $d_{x^{2}-y^{2}}$-wave gap. We also find that the inclusion of a higher harmonic basically doesn't alter the local electronic structure near a non-magnetic impurity; in contrast, with introducing AF SDW order, the LDOS presents spin-resolved feature, i.e. a degenerate single resonance state at the impurity site separates into two resonance states due to the different spin component induced by the presence of AF SDW order. Thus we strongly suggest that the future spin-polarized STM measurements should be performed to differentiate two above scenarios and shed light on the real physical origin of non-monotonic $d_{x^{2}-y^{2}}$-wave gap in electron-doped cuprate superconductors.

We thank Prof. I. Eremin, Prof. Xi Dai for the careful reading of the manuscript and fruitful discussions.
* Current address: Beijing National Laboratory for Condensed Matter Physics, and Institute of Physics, Chinese Academy of Sciences, Beijing 100190, China.

1 P. Krotkov, and A.V. Chubukov, Phys. Rev. Lett. 96, 107002 (2006); Phys. Rev. B 74, 014509 (2006).

2 D. Manske, I. Eremin, and K.-H. Bennemann, Phys. Rev. B 62, 13922 (2000).

${ }^{3}$ L. Alff et al., Phys. Rev. Lett. 83, 2644 (1999); S. Kashiwaya et al., Phys. Rev. B 57, 8680 (1998).

4 A. Biswas et al., Phys. Rev. Lett. 88, 207004 (2002); J.A. Skinta et al., Phys. Rev. Lett. 88, 207005 (2002).

${ }^{5}$ C.C. Tsuei, and J.R. Kirtley, Phys. Rev. Lett. 85, 182 (2000).

6 Guo-qing Zheng et al., Phys. Rev. Lett. 90, 197005 (2003).

7 H. Matsui et al., Phys. Rev. Lett. 95, 017003 (2005).

8 N.P. Armitage et al., Phys. Rev. Lett. 86, 1126 (2001).

9 G. Blumberg et al., Phys. Rev. Lett. 88, 107002 (2002).

${ }^{10}$ H. Yoshimura and D.S. Hirashima, J. Phys. Soc. Jpn. 73,
2057 (2004); J. Phys. Soc. Jpn. 74, 712 (2005).

11 Q.S. Yuan, F. Yuan, and C.S. Ting, Phys. Rev. B 73, 054501 (2006); Phys. Rev. B 74, 214503 (2006).

12 H.J. Kang et al., Nature 423, 522 (2003); H.J. Kang et al., Phys. Rev. B 71, 214512 (2005).

13 A.N. Lavrov et al., Phys. Rev. Lett. 92, 227003 (2004); W. Yu et al., Phys. Rev. B 76, 020503 (2007).

14 N.P. Armitage et al., Phys. Rev. Lett. 88, 257001 (2002).

15 H.G. Luo, and T. Xiang, Phys. Rev. Lett. 94, 027001 (2005).

16 D.K. Morr, Phys. Rev. Lett. 89, 106401 (2002); Q.H. Wang, Phys. Rev. Lett. 88, 057002 (2002); A.V. Balatsky, I. Vekhter, and Jian-Xin Zhu, Rev. Mod. Phys 78, 373 (2006).

17 L. Yu, Acta. Phys. Sin 21, 75 (1965); H. Shiba, Prog. Theor. Phys 40, 435 (1968); A.I. Rusinov, Sov. Phys. JETP 29, 1101 (1969).

18 Bin Liu, and I. Eremin, Phys. Rev. B 78, 014518 (2008). 
19 Tanmoy Das, R.S. Markiewicz, and A. Bansil, Phys. Rev. B 74, 020506(R) (2006).

${ }^{20}$ Bin Liu, and Ying Liang, Phys. Rev. B 77, 245121 (2008).
21 Y. Dagan, R. Beck, and R.L. Greene, Phys. Rev. Lett. 99, 147004 (2007). 